\title{
Synthesis and antimicrobial activity of some new imidazolinone derivatives containing benzimidazole
}

\author{
Prakash Mehta ${ }^{1}$, Prakash Davadra ${ }^{2}$, Nirav Shah ${ }^{2}$, Hitendra Joshi ${ }^{2, *}$ \\ ${ }^{1}$ Home Science Department, Dr. Subhash Mahila Arts, Commerce \& Home Science College, \\ Junagadh - 362 001, Gujarat, India \\ ${ }^{2}$ Department of Chemistry, Saurashtra University, Rajkot - 360 005, Gujarat, India \\ *E-mail address: drhsjoshi49@gmail.com
}

\begin{abstract}
In this study some new o-benzimidazol-2'-yl-benzamido-p'-benzamido-2-phenyl-4-substituted phenyl-5-oxo-Imidazolines 3a-3j were synthesized. To synthesized target molecules we used various substituted oxazolone derivatives, synthesized from substituted benzaldehyde with hippuric acid. Substituted oxazolones $\mathbf{2} \mathbf{a - 2} \mathbf{j}$ were reacted with carbohydrazide derivative of benzimidazole $\mathbf{1}$ in presence of pyridine as base to obtained substituted imidazolinone derivatives. All synthesized compounds were characterized by IR, ${ }^{1} \mathrm{H}$ NMR, elemental analysis and further supported by mass spectroscopy. All synthesized compounds were screened for their antimicrobial activity against gram positive and gram negative bacteria which showed moderate to good activity. All compounds showing good to moderate active against fungal strain as compare to standard drug.
\end{abstract}

Keywords: Benzimidazole; imidazolinone; antimicrobial activity

\section{INTRODUCTION}

In recent years, organic research for synthesis of novel nitrogen containing heterocyclic ring system is emerging. Over the years of active research, benzimidazole is reported to shown wide range of therapeutic activities [1-7]. Albendazole, mebendazole, thiabendazole as antihelmintics; omeprazole, lansoprazole, pantoprazole as proton pump inhibitors; astemizole as antihistaminic; enviradine as antiviral; candesarten cilexitil and telmisartan as antihypertensive which contains benzimidazole as active pharmacophore. Imidazolinone ring system is also extensively research for biological as well as chemical aspects since long time. The imidazolinones are associated with a wide range of therapeutic activities like fungicidal, herbicidal, and vasodilator and anticancer etc. [8-13]. The basic imidazole nucleus, present in azalactone containing oxazolone moiety, is of great importance for generating penicillin type of drug intermediates and synthetic hormonal compounds. Oxoimidazolines have been reported to exhibit antibacterial [14,15], antifungal [16] and antimicrobial activities [17-24]. The therapeutic importance of the compounds inspired us to synthesize some potential imidazolinones containing benzimidazole as scaffold. 


\section{EXPERIMENTAL}

All chemicals and solvents were purchased from Spectrochem Pvt Ltd., Mumbai of AR grade and were used without further purification. Melting points were taken in open capillary method and are uncorrected. IR spectra were recorded on FTIR-8400 spectrophotometer (Shimadzu, Kyoto, Japan), using DRS prob. KBr pallet. ${ }^{1} \mathrm{H}-\mathrm{NMR}$ spectra of the synthesized compounds were recorded on a Bruker-Avance-II $(400 \mathrm{MHz})$ DMSO-d 6 solvent. Chemical shifts are expressed in $\delta$ ppm downfield from TMS as an internal standard. Mass spectra were determined using direct inlet probe on a GCMS-QP 2010 mass spectrometer (Shimadzu, Kyoto, Japan). Physical constants of the synthesized compounds $\mathbf{3 a - 3 j}$ are shown in Table 1.

\section{1. Procedure for synthesis of $2-(1 \mathrm{H}-B$ enzimidazol-2-yl)-N-(4-hydrazinocarbonyl- phenyl) benzamide (1)}

Step 1: An equimolar amount of pthalic anhydride and o-phenylene diamine were taken in $\mathrm{RBF}$ Direct heat the reaction mass at $140-150{ }^{\circ} \mathrm{C}$ to obtained o-Benzoylene 2-1benzimidazole [18].

Step 2: A mixture of o-benzoylene 2-1-benzimidazole $(1 \mathrm{mmol})$ and benzocaine $(1 \mathrm{mmol})$ were refluxed for $4-5$ hours in DMF at $150{ }^{\circ} \mathrm{C}$. Completion of reaction was monitored by TLC. Pour the reaction in chilled water and filter out the precipitate of crude product. Dry in vacuo. Crystalline from DMSO to obtained analytical grade pure 2-o-(4'-carbethoxyphenyl amino carbonyl phenyl) benzimidazole. Yield $85 \%$.

Step 3: In ethanolic solution of 2-o(4'-carbethoxyphenyl amino carbonyl phenyl)benzimidazole $(1 \mathrm{mmol})$, hydrazine hydrate $(10 \mathrm{mmol})$ was added and reflux overnight. Cool down the reaction and filter the precipitate product. Wash the product with chilled ethanol to collect the analytical pure grade 2-(1H-Benzimidazol-2-yl)-N-(4-hydrazinocarbonyl-phenyl) benzamide. Yield $80 \%$.<smiles>Nc1ccccc1N</smiles>

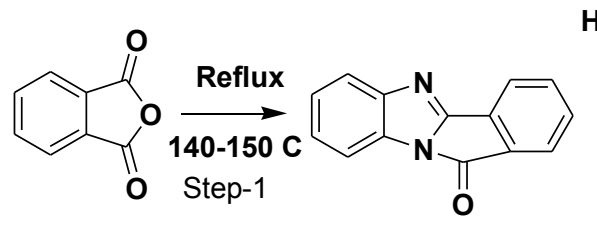

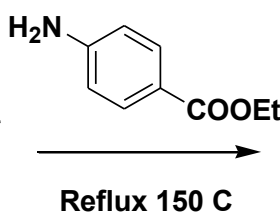

Step- 2<smiles>CCOC(=O)c1ccc(NC(=O)c2ccccc2-c2nc3ccccc3[nH]2)cc1</smiles><smiles>NNC(=O)c1ccc(NC(=O)c2ccccc2-c2nc3ccccc3[nH]2)cc1</smiles>

Scheme I

(1)

\section{2. General process for synthesis of oxazol-5(4H)-ones (2a-2j) [Erlenmeyer azlactone synthesis]}

A mixture substituted benzaldehyde $(1 \mathrm{mmol})$, hippuric acid $(1 \mathrm{mmol})$ and potassium acetate $(1 \mathrm{mmol})$ in acetic anhydride $(2.5 \mathrm{mmol})$ (Scheme II) was refluxed with stirring for 15 
min. (reaction progress was monitored by TLC). The mixture was then cooled down and neutralized by addition of solid potassium carbonate. The solid product was separated by filtration, dried and purified by crystallization.

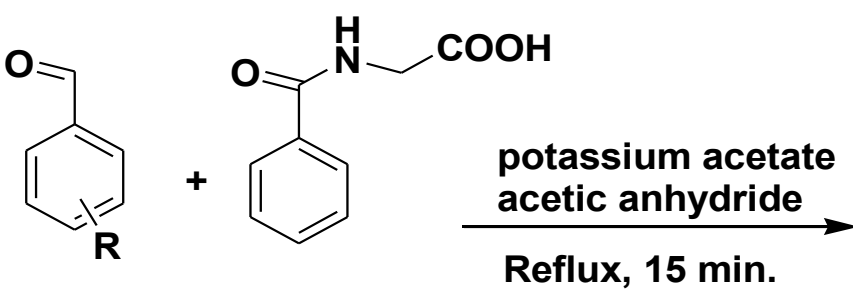

Reflux, 15 min.<smiles>[R]c1ccc(/C=C2\N=C(c3ccccc3)OC2=O)cc1</smiles>

$(2 a-j)$

Scheme II

\section{3. General procedure for the synthesis of o-benzimidazol-2'-yl-benzamido-p'-} benzamido-2-substituted phenyl-4-substituted phenyl-5-oxo-Imidazolines (3a-j)

A mixture of compound $\mathbf{1}(1 \mathrm{mmol})$ and substituted compound $\mathbf{2 a - 2 \mathbf { j }}(1 \mathrm{mmol})$ was placed in a round bottom flask and $10 \mathrm{ml}$ of pyridine were added to it. The reaction mixture was refluxed in oil bath (Scheme III). The progress of the reaction was checked by TLC. After completion of the reaction, reaction mass was poured into ice-cold water and then a required amount of conc. hydrochloric acid was added to neutralize the reaction mixture. The solid obtained was left overnight, filtered and washed with water. The product was dried and recrystallized from DMSO.<smiles>NNC(=O)c1ccc(NC(=O)c2ccccc2-c2nc3ccccc3[nH]2)cc1</smiles>

(1)<smiles>[R][X]c1ccc(/C=C2\N=C(c3ccccc3)OC2=O)cc1</smiles>

$(2 \mathrm{a}-\mathrm{j})$

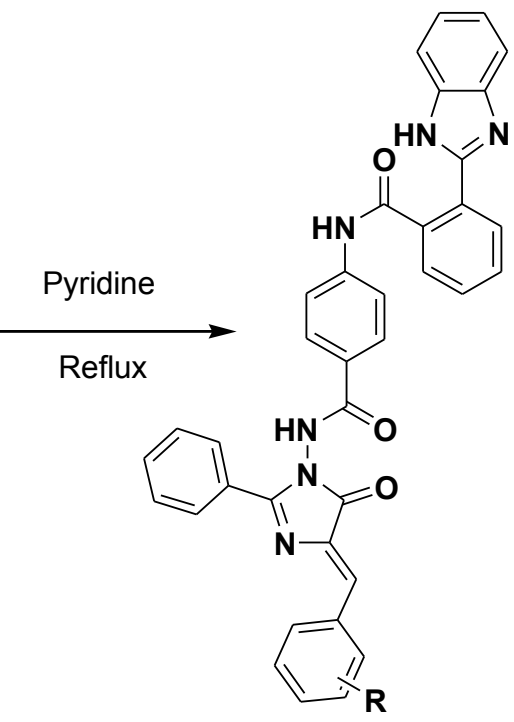

\section{Scheme III}

\section{4. Characterization of imidazolinone derivative}

Compound 3f: IR (KBr) v(cm $\left.{ }^{-1}\right): 3290$ (N-H str.), 3040 (C-H str.), 2970 (C-H str.), 2873 (C-H str.), 1620 (C=C str.), 1455 (C-H bend.), 1375 (C-H bend.), 1310 (C-N str.), 1220 (C-H def.), 1140 ( C-N-C str.), 1020 (C-O-C str.), 1060 (C=O str.), 810 (C-H def. bend.), ${ }^{1} \mathbf{H}$ NMR (DMSO-D $)_{6} \boldsymbol{\delta} ; 8.2(1 \mathrm{H}, \mathrm{s},-\mathrm{NH}), 6.8-8.1(21 \mathrm{H}, \mathrm{m}, \mathrm{Ar}-\mathrm{H}), 6.2(1 \mathrm{H}, \mathrm{s},-\mathrm{CH}=), 3.9(3 \mathrm{H}, \mathrm{s},-$ 
$\left.\mathrm{OCH}_{3}\right), \mathbf{M}^{+}(\mathrm{m} / \mathrm{z})=632$. Elemental analysis: Calculated $\mathrm{C}(72.14 \%) \mathrm{H}(4.46 \%) \mathrm{N}(13.28$ \%), Obtained : C (72.05\%), H (4.42\%), N (13.21\%).

Table 1. Physical constant of o-Benzimidazol-2'-yl-benzamido-p'-benzamido-2-phenyl-4-substituted phenyl-5-oxo-imidazolines derivatives (3a-3j).

\begin{tabular}{|c|c|c|c|c|c|c|}
\hline No & Comp. & $\mathbf{R}$ & $\begin{array}{c}\text { Molecular } \\
\text { Formula }\end{array}$ & $\begin{array}{c}\text { Molecular } \\
\text { Weight }\end{array}$ & $\begin{array}{c}\text { Yield } \\
(\mathbf{\%})\end{array}$ & $\begin{array}{c}\text { M.P. } \\
\left({ }^{\circ} \mathbf{C}\right)\end{array}$ \\
\hline 1 & $3 \mathrm{a}$ & $\mathrm{H}$ & $\mathrm{C}_{37} \mathrm{H}_{26} \mathrm{~N}_{6} \mathrm{O}_{3}$ & 602 & 60 & 238 \\
\hline 2 & $3 \mathrm{~b}$ & $4-\mathrm{NH}_{2}$ & $\mathrm{C}_{37} \mathrm{H}_{27} \mathrm{~N}_{7} \mathrm{O}_{3}$ & 617 & 75 & 241 \\
\hline 3 & $3 \mathrm{c}$ & $2-\mathrm{Cl}$ & $\mathrm{C}_{37} \mathrm{H}_{25} \mathrm{ClN}_{6} \mathrm{O}_{3}$ & 637 & 70 & 212 \\
\hline 4 & $3 \mathrm{~d}$ & $4-\mathrm{Cl}$ & $\mathrm{C}_{37} \mathrm{H}_{25} \mathrm{ClN}_{6} \mathrm{O}_{3}$ & 637 & 78 & 234 \\
\hline 5 & $3 \mathrm{e}$ & $3,4-\mathrm{di} \mathrm{OCH}$ & $\mathrm{C}_{34} \mathrm{H}_{28} \mathrm{~N}_{6} \mathrm{O}_{5}$ & 600 & 82 & 252 \\
\hline 6 & $3 \mathrm{f}$ & $4-\mathrm{OCH}$ & $\mathrm{C}_{38} \mathrm{H}_{28} \mathrm{~N}_{6} \mathrm{O}_{4}$ & 632 & 55 & 267 \\
\hline 7 & $3 \mathrm{~g}$ & $2-\mathrm{furyl}$ & $\mathrm{C}_{30} \mathrm{H}_{22} \mathrm{~N}_{6} \mathrm{O}_{4}$ & 530 & 70 & 198 \\
\hline 8 & $3 \mathrm{~h}$ & $2-\mathrm{OH}$ & $\mathrm{C}_{32} \mathrm{H}_{24} \mathrm{~N}_{6} \mathrm{O}_{4}$ & 556 & 77 & 202 \\
\hline 9 & $3 \mathrm{i}$ & $4-\mathrm{OH}$ & $\mathrm{C}_{32} \mathrm{H}_{24} \mathrm{~N}_{6} \mathrm{O}_{4}$ & 556 & 60 & 217 \\
\hline 10 & $3 \mathrm{j}$ & $4-\mathrm{Br}$ & $\mathrm{C}_{32} \mathrm{H}_{23} \mathrm{BrN}_{6} \mathrm{O}_{3}$ & 619 & 58 & 245 \\
\hline
\end{tabular}

\section{ANTIMICROBIAL ACTIVITY}

The MICs of synthesized compounds were carried out by broth micro dilution method as described by Rattan 26]. Antibacterial activity was screened against two gram positive (Bacillus megaterium, Streptococcus citreus) and two gram negative (Escherichia coli, Salmonella typhosa) bacteria. Ampicillin, norfloxacacin and chloramphenicol were used as a standard antibacterial agent.

Antifungal activity was screened against Aspergillus Niger. Greseofulvin was used as a standard antifungal agent. The obtained results for compounds $\mathbf{3 a - 3} \mathbf{j}$ are recorded in Table 2. 
Table 2. Antimicrobial activity of imidazolinone derivatives (3a-3j).

\begin{tabular}{|c|c|c|c|c|c|c|}
\hline \multirow{2}{*}{ Compound } & \multirow{2}{*}{$\mathbf{R}$} & \multicolumn{4}{|c|}{ Antibacterial activity } & \multirow{2}{*}{$\begin{array}{c}\text { Antifungal } \\
\begin{array}{c}\text { activity } \\
(\%)\end{array} \\
\begin{array}{c}\text { A. } \\
\text { niger }\end{array}\end{array}$} \\
\hline & & $\begin{array}{c}\text { S. } \\
\text { aureus }\end{array}$ & $\begin{array}{c}\text { S. } \\
\text { epidermidis }\end{array}$ & $\begin{array}{l}\text { E. } \\
\text { coli }\end{array}$ & $\begin{array}{c}\mathrm{P} . \\
\text { aeruginosa }\end{array}$ & \\
\hline $3 a$ & $\mathrm{H}$ & 80 & 33 & 50 & 67 & 50 \\
\hline $3 b$ & $4-\mathrm{NH}_{2}$ & 70 & 71 & 55 & 67 & 63 \\
\hline $3 \mathrm{c}$ & $2-\mathrm{Cl}$ & 60 & 54 & 86 & 48 & 67 \\
\hline $3 d$ & $4-\mathrm{Cl}$ & 68 & 69 & 68 & 81 & 83 \\
\hline $3 \mathrm{e}$ & $\begin{array}{l}3,4-\mathrm{di} \\
\mathrm{OCH}_{3}\end{array}$ & 85 & 83 & 45 & 89 & 46 \\
\hline $3 f$ & $4-\mathrm{OCH}_{3}$ & 75 & 54 & 85 & 86 & 38 \\
\hline $3 g$ & 2-furyl & 90 & 38 & 77 & 80 & 54 \\
\hline $3 \mathrm{~h}$ & $2-\mathrm{OH}$ & 50 & 79 & 55 & 52 & 75 \\
\hline $3 \mathrm{i}$ & $4-\mathrm{OH}$ & 45 & 42 & 64 & 67 & 71 \\
\hline $3 \mathrm{j}$ & $4-\mathrm{Br}$ & 80 & 70 & 65 & 78 & 72 \\
\hline Ampicillin & - & 100 & 100 & 100 & 100 & - \\
\hline Norfloxacin & - & 100 & 100 & 100 & 100 & - \\
\hline $\begin{array}{l}\text { Chloramphen } \\
\text { icol }\end{array}$ & - & 100 & 100 & 100 & 100 & - \\
\hline Greseofulvin & - & - & - & - & - & 100 \\
\hline
\end{tabular}

\section{CONCLUSION}

In present report, we synthesized new carbohydrazide derivative of benzimidazole from very cheap starting material. Synthesis of some new imidazolinone derivatives (3a-3j) were carried out by reaction of carbohydrazide $\mathbf{1}$ with substituted oxazolone derivatives $(\mathbf{2} \mathbf{a}-\mathbf{2} \mathbf{j})$. 
All synthesized compounds were obtained in good to moderate yield. The synthesized compounds were characterized by ${ }^{1} \mathrm{H}$ NMR, Mass and IR spectroscopy and the obtained results are showing good agreement with the synthesized structures. From the results of antimicrobial data, compounds $\mathbf{3} \mathbf{j}$ containing $4-\mathrm{Br}$ group is most active for antimicrobial activity as compared to others.

\section{References}

[1] Q. McKellar, E. Scott, J. Vet. Pharmacol. Ther. 13 (1990) 223.

[2] A. Spasov, I. Yozhitsa, L. Bugaeva, V. Anisimova, Pharm. Chem. J. 33 (1999) 232.

[3] J. Rossignol, H. Maisonneuve, Ann. Trop. Med. Parasitol. 78 (1984) 135.

[4] A. Patil, S. Ganguly, S. Surana, Rasayan J. Chem. 1 (2008) 447.

[5] A. Dubey, P. Sanyal, Online Vet. J. 5 (2010) 63.

[6] M. Boiani, M. Gonzalez, Mini Rev. Med. Chem. 5 (2005) 409.

[7] B. Narasimhan, D. Sharma, P. Kumar, Med. Chem. Res. 21 (2012) 269.

[8] Duschinsky R. Imidazolone derivatives. US patent. 1995 US2707186.

[9] D. Naithani, V. Srivastava, J. Barthwal, A. Saxena, T. Gupta, K. Shanker, Indian J Chem. 28B (1989) 990.

[10] V. Kachhadia, M. Patel, H. Joshi, J. Serb. Chem. Soc. 70 (2005) 153.

[11] M. Ding, G. Zeng G, Z. Liu, Phosphorus, Sulfur Silicon Relat. Elem. 177 (2002) 1315.

[11] X. Huang, Z. Liu, F. Yang, M. Ding, Phosphorus, Sulfur Silicon Relat. Elem. 182 (2007) 939.

[12] S. Demirayak, S. A. Karaburun, I. Kayagil, K. Erol, B. Sirmagul, Arch Pharm Res. 27 (2004) 13.

[13] K.Thaker, P. Zalavadia, H. Joshi, Jour. of Science Islamic Repb. of Iran 16 (2005) 139.

[14] A. Solankee, S. Solankee, G. Patel, Rasayan J Chem. 1 (2008) 228.

[15] El-Sayed Ali T., Abdel-Aghfaar Abdel-Aziz S., Metwali El-Shaaer H., Ismail Hanafy F., Zaky El-Fauomy A., Turk J Chem. 32 (2008) 365.

[16] R. Mistry, K. Desai, Eur Jour. Chem. 2 (2005) 42.

[17] A. Bistrzycki, A. Lecco, Helv. Chim. Acta. 4 (1921) 427.

[18] Rattan A. Antimicrobials in laboratory medicine. 1st ed. New Delhi: Churchill Livingstone (2000) 85.

[19] Rahul R. Tripathi, Ratnamala P. Sonawane, International Letters of Chemistry, Physics and Astronomy 10(2) (2013) 119-125.

[20] Mallikarjun S. Yadawe, Shrishila N. Unki, Sangamesh A. Patil, International Letters of Chemistry, Physics and Astronomy 12 (2013) 94-104. 
[21] G. Thirunarayanan, International Letters of Chemistry, Physics and Astronomy 5 (2014) 89-98.

[22] V. J. Faldu, P. K. Talpara, N. H. Bhuva, P. R. Vachharajani, V. H. Shah, International Letters of Chemistry, Physics and Astronomy 6 (2014) 26-32.

[23] Piyush B. Vekariya, Jalpa R. Pandya, Vaishali Goswami, Hitendra S. Joshi, International Letters of Chemistry, Physics and Astronomy 7 (2014) 45-52.

[24] R. G. Vaghasiya, H. B. Ghodasara, P. R. Vachharajani, V. H. Shah, International Letters of Chemistry, Physics and Astronomy 8 (2014) 30-37. 